\title{
Volar Distal Radius Anatomy Applied to the Treatment of Distal Radius Fracture
}

\author{
Junya Imatani, MD, $\mathrm{PhD}^{1}$ Keiichi Akita, $\mathrm{MD}, \mathrm{PhD}^{2}$ \\ ${ }^{1}$ Department of Orthopaedic Surgery, Okayama Saiseikai General \\ Hospital, Okayama, Japan \\ 2 Department of Clinical Anatomy, Graduate School Tokyo Medical \\ and Dental University, Bunkyo-ku, Tokyo, Japan
}

\begin{abstract}
Address for correspondence Junya Imatani, MD, PhD, Department of Orthopaedic Surgery, Okayama Saiseikai General Hospital, 2-25 Kokutaicho Kita-ku, Okayama, 700-8511, Japan (e-mail: imatani@saiseidr.jp).
\end{abstract}

J Wrist Surg 2017;6:174-177.

\begin{abstract}
Keywords

- distal radius fracture

- anatomy

- volar locking plate

Volar locking plate (VLP) fixation has become the standard surgical treatment for distal radius fractures. However, flexor tendon rupture is one of the major complications following volar plating. This detailed review on the anatomy and morphology of the volar distal radius might facilitate appropriate placement of the volar plate and thereby avoid flexor tendon rupture. We introduce safe and secure VLP fixation along standard surgical procedures to avoid complications based on anatomy of the volar surface of the distal radius.
\end{abstract}

Volar locking plate (VLP) fixation has become the standard surgical technique for unstable distal radius fractures (DRFs). Flexor tendon rupture is one of the major complications after volar plating. The published studies on distal radius anatomy have focused on distal limit and watershed line should be taken into account to reduce the incidence of flexor tendon attritional injury during volar plating of distal radius.

\section{The Watershed Line Concept}

Windisch et $\mathrm{al}^{1}$ were the first to describe the area where the capsule inserts between two lines and two contours on the volar aspect of the distal radius. They also reported a protuberance, which they termed the promontory of the radius. $^{2}$ In 2006, Orbay and Touhami ${ }^{3}$ reported that the concave surface of the volar radius was limited distally by a transverse ridge or watershed line. Implants placed over or projecting above the watershed line can potentially irritate the flexor tendons and finally rupture them. Gasse et $\mathrm{al}^{4}$ confirmed the presence of these two easily recognizable lines in 70 distal radius specimens from cadavers; the ulnar column was found to be longer distally than the radial column, and the radial styloid process was not within the distal radius plane.

received

May 12, 2017

accepted after revision

May 25, 2017

published online

June 27, 2017

\section{Macroscopic Characteristics of Volar Distal Radius}

We investigated the macroscopic appearance of the volar aspect of the distal radius in 20 cadaver specimens to clarify the relationships of the distal radius, the volar radiocarpal ligaments, and the pronator quadratus. ${ }^{5,6}$ In the ulnar aspect, the volar radius had two main distal transverse lines indicating the bony prominence. One comprised the distal higher line (bold black dotted line in -Fig. 1) and the other comprised the proximal lower line forming the distal bony ridge of the pronator fossa (thin black dotted line in - Fig. 1). In the radial aspect, these two lines merged. The volar radius also had ulnar and radial bony prominences (white and black dots in - Figs. 1 and 2 ) on the distal higher line, which were clearly identified in 20 specimens even on the articular capsule and radiocarpal ligaments. The ulnar bony prominence was larger than the radial bony prominence and it was the highest point on the volar aspect of the distal end of the radius. A line connecting the distal margin of the pronator quadratus (small thin black dotted line in - Fig. 1) did not correspond to the distal ridge of the pronator fossa. We also showed that the flexor digitorum profundus (FDP)-II tendon of the second finger ran on the lateral surface of the ulnar prominence of the volar distal end of radius. ${ }^{7}$ The flexor

Copyright @ 2017 by Thieme Medical Publishers, Inc., 333 Seventh Avenue, New York, NY 10001, USA. Tel: +1(212) 584-4662.
Dol https://doi.org/ 10.1055/s-0037-1603995. ISSN 2163-3916. 


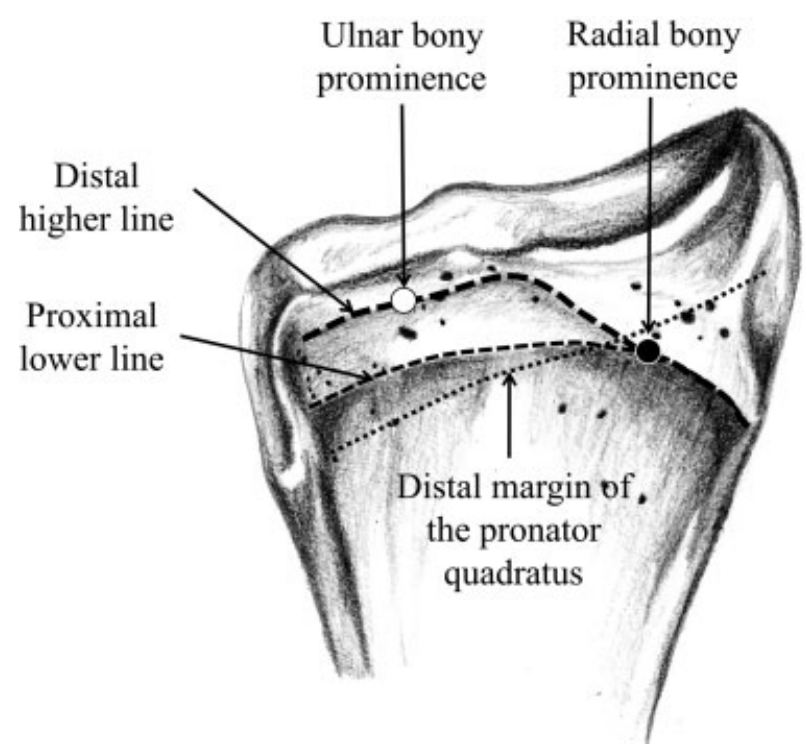

Fig. 1 Schematic drawing of the volar aspect of radius based on our macroscopic findings. Two dotted lines (bold and thin black) indicate the bony inflexion points based on the macroscopic and microscopic observations. The bold black dotted line indicates the distal higher bony inflexion points and the thin black dotted line indicates the proximal lower bony inflexion points as the most distal ridge of pronator fossa. The small thin black dotted line indicates the distal margin of the pronator quadratus muscle. Two bony prominences, ulnar and radial, on the bold black dotted line are indicated by white and black dots, respectively.

pollicis longus (FPL) tendon ran just lateral to the FDP-II tendon in all 26 specimens in this study. ${ }^{7}$ Therefore, the ulnar bony prominence (white dot in - Fig. 2) was recommended to be a good landmark of the distal limit for safe plate positioning (the safe zone). The dangerous zone of flexor impingement was on average $11 \pm 1 \mathrm{~mm}$ radial to the ulnar bony prominence of the volar distal end of the radius. In the

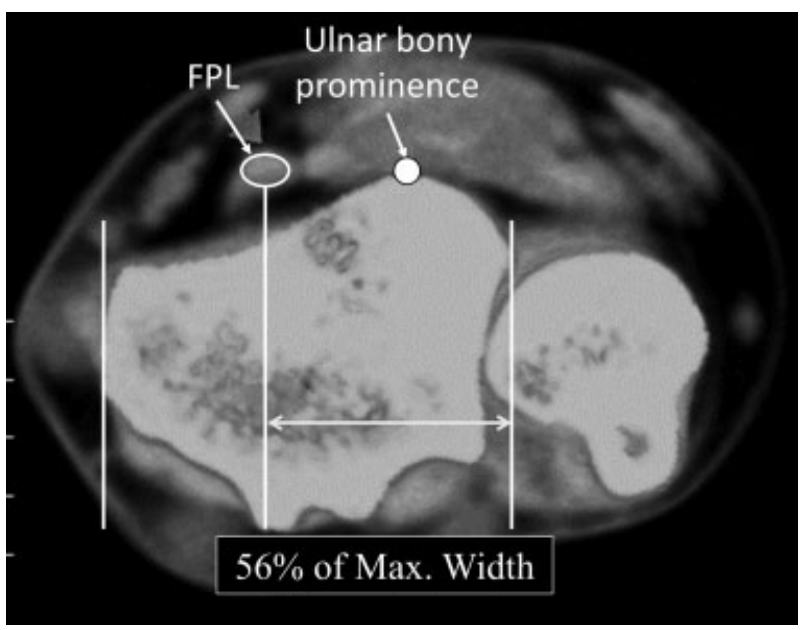

Fig. 3 Multi-detector computed tomography (MDCT) evaluation of the anatomical relationship between the flexor pollicis longus (FPL) and the bony prominences of the volar distal radius. The FPL tendon ran through from the ulnar corner of the distal radius in $56 \%$ of the 50 normal wrist joints.

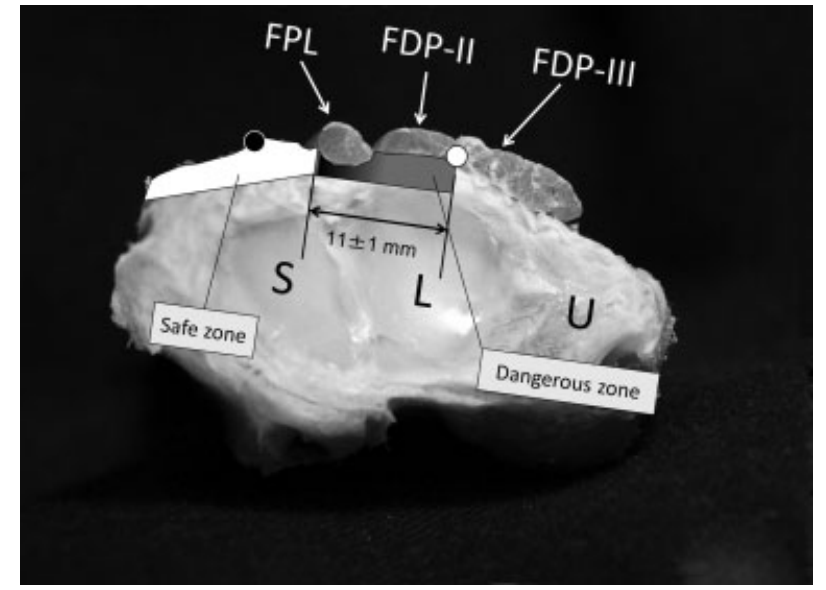

Fig. 2 Articular view from the distal aspect. The radial bony prominence is indicated by the black dot and the ulnar bony prominence is indicated by the white dot. The flexor digitorum profundus (FDP)-II tendon of the second finger runs on the lateral surface of the ulnar prominence. In all 26 specimens, the flexor pollicis longus (FPL) tendon ran immediately lateral to the FDP-II tendon and between the two bony prominences. The dangerous zone is on average $11 \pm 1 \mathrm{~mm}$ radial to the ulnar bony prominence of the radius. In this zone, there should not be protrusion of volar implant anterior to the rim of the distal radius. S, scaphoid bone; L, lunate bone; $\mathrm{U}$, ulna.

dangerous zone, volar implants should not protrude anterior to the rim of the distal radius ${ }^{6}$ ( - Fig. 2). The multidirectional computer tomography study examining the anatomical relationship between the flexor tendons and the bony prominences in 50 normal wrist joints ${ }^{8}$ indicated that the FPL tendon ran from the ulnar corner of the distal radius in $56 \%$ of cases (-Fig. 3). Limthongthang et $\mathrm{al}^{9}$ also used a computer modeling to study the location of the FPL tendon. At the watershed line, the FPL was located at $54 \%$ of the maximal width of the radius as measured from its volar ulnar corner.

\section{Microscopic Analysis of Volar Distal Radius}

The serial sagittal histological sections of the wrist regions demonstrated the positional relationship of the volar aspect of the distal radius, pronator quadratus, intermediate fibrous zone, and radiocarpal ligaments ${ }^{5}$ in - Fig. 4 . The distal end of the volar radius was classified into two general parts: the radial and ulnar halves. In the ulnar half ( - Figs. 4A, 4B, 4C), two bony demarcation points were observed on the volar surface of the distal end of the radius: the proximal point (indicated by the asterisks) and the distal point (indicated by the black dots). In the radial half ( - Figs. 4D, 4E), one bony demarcation point (indicated by the asterisks) was observed in each section. In the ulnar section of the lateral half ( $\mathbf{F i g}$. 4D, 4E), the two demarcation points overlapped. In these histological sections, the watershed line might not be a distinct line and corresponded to the distal margin of the pronator fossa in the radial aspect of the volar radius and to a hypothetical line between the distal higher and proximal lower lines in the ulnar aspect. The radial and ulnar bony prominences on the volar radius should be considered as key structures for accurate plate placement to avoid flexor tendon injury. 
A

B

C

D

$\mathrm{E}$

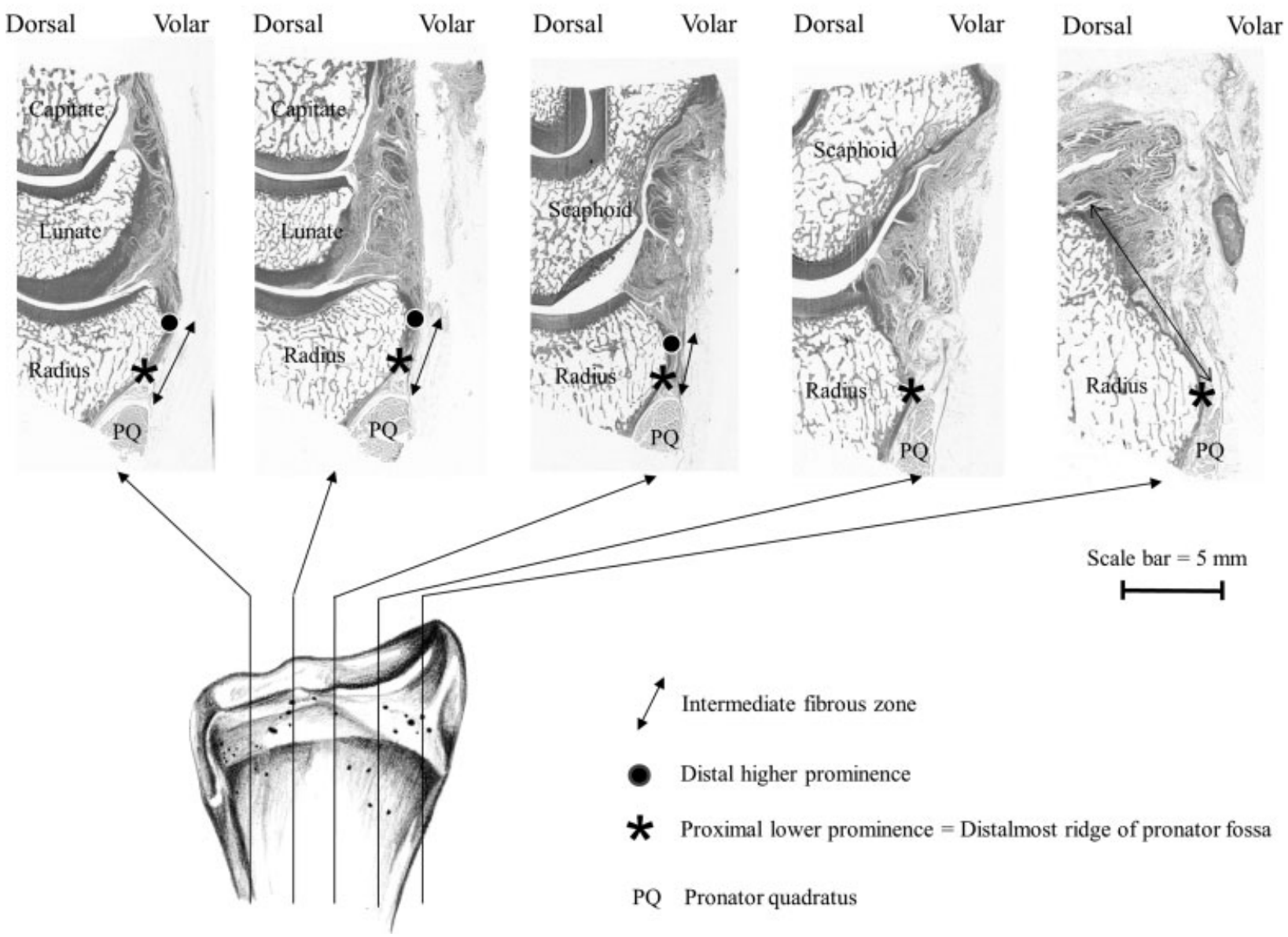

Fig. 4 Sagittal sections of the distal end of the radius and volar wrist region. The black dots indicate the distal higher prominence and the asterisks indicate the proximal lower prominence as the most distal ridge of the pronator fossa. The black double arrow indicates the intermediate fibrous zone between the radiocarpal ligament and PQ. PQ, pronator quadratus muscle.

\section{Volar Morphology of the Distal Radius: A Quantitative Analysis}

Oura et $\mathrm{al}^{10}$ observed the anatomical shape of the volar surface of the distal radius and the location of the FPL tendon based on computed tomography (CT) scans of 70 normal forearms examined with the axial crosssectional views with $2 \mathrm{~mm}$ intervals. The volar surface of the distal radius was concave in the axial plane in all the forearms. The radial longitudinal deficiency (RLD), which is the maximum depth from line $\mathrm{L}$ to the volar surface of the radius at the level of the volar edge of the lunate fossa was $1.3 \pm 0.6 \mathrm{~mm}$. This value increased gradually, reaching the maximum $(1.7 \pm 0.8 \mathrm{~mm})$ at $6 \mathrm{~mm}$ from the origin and then progressively decreased toward the proximal radius. The FPL was closest to the radius at $2 \mathrm{~mm}$ proximal to the palmar edge of the lunate fossa and the volar surface of the distal radius was supinated from proximal to distal ( $\theta$ angle: average amount of external rotation was $10.1 \pm 3.4$ degrees, - Fig. 5).

In a quantitative analysis of 200 standardized lateral wrist radiographs by Yoneda et $\mathrm{al}^{11}{ }^{11}$ the authors reported the teardrop height ratio (RTH) and the teardrop inclination angle
(TIA) representing the volar projection of the lunate facet of the distal radius to be $0.42(0.30-0.56)$ and 28.8 degrees (9.9-44.9 degrees), respectively (-Fig.6). They analyzed the influence of the teardrop morphology by analyzing the fit of the three

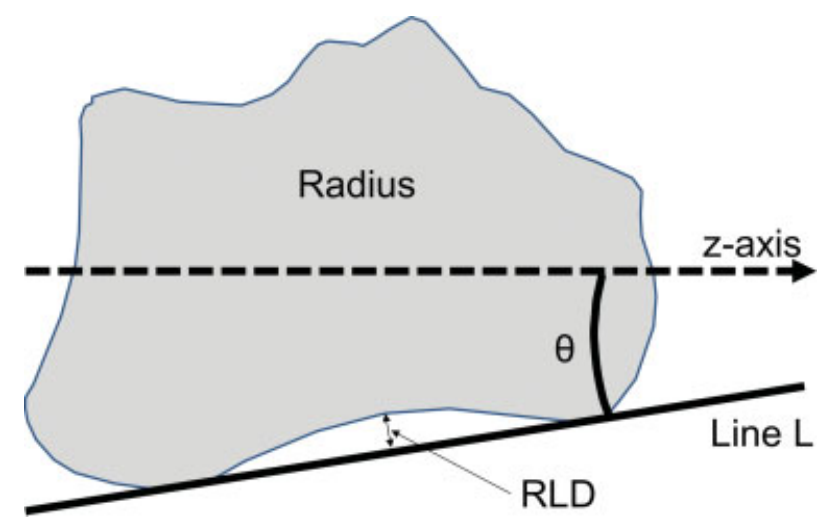

Fig. 5 Crosssections of the radius. Line $L$ is tangent to the edges of the concavity of the volar radius. The dotted line represents the z-axis; $R L D$ represents maximum depth from line $L$ to the volar surface of the radius; and $\theta$ represents angle between line $L$ and the $z$-axis. 


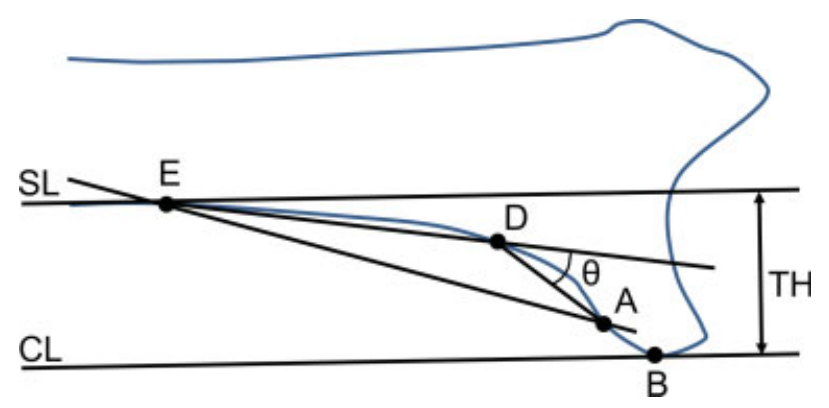

Fig. 6 A shaft line (SL, along the volar aspect of the radius shaft) and critical line ( $\mathrm{CL}$, tangential to the most volar extent of the teardrop) are drawn on the ulnar aspect. The distance between the critical and shaft lines is measured as the teardrop height (TH). On the ulnar cortical line, the proximal point of the curve on the SL (point $E$ ), proximal bony demarcation point (point $A$ ), and point farthest from line $A E$ (point $D$ ) are determined. The teardrop inclination angle (TIA) is measured as the external angle made by the two, line segments, DE and AD. The ratio of the teardrop height (RTH) was calculated by dividing the TH from the maximum width of the radius through the top of Lister's tubercle on the lateral radiograph.

locking plates to three radii with differing teardrop inclination angles using a three-dimensional (3D) computer-aided design system. They also investigated the interindividual variation in the shape of the teardrop and its influence on the fit of the volar plate, highlighting the importance of careful plate selection to achieve osteosynthesis of bones with a high teardrop inclination angle (-Fig. 6).

Pichler and colleagues ${ }^{12}$ measured the profile of the volar distal radius of 100 cadaver specimens with a common profile gauge. The mean circular arc radius of the distal volar surface was $2.6 \mathrm{~cm}$ on the radial side and $2.3 \mathrm{~cm}$ on the ulnar side. In $37 \%$ of these cases, the circular arc radius flattened toward the ulnar side while in $63 \%$, it flattened toward the radial side. They concluded that these characteristics may lead to malrotation of the distal fragment following VLP fixation of DRFs. An inadequate plate position may result in the flexor tendon irritation due to the discrepancy between the plate radius curvature and the radius curvature of either the radial or ulnar aspect of the distal radius.

Andermahr et al $^{13}$ measured 48 distal radius specimens using 3D-CT scans to quantify the anatomical details of the volar part of the lunate facet of the distal radius. They observed that the volar lunate facet projected approximately $3 \mathrm{~mm}$ (or 16\% of the dorsal-volar height of the lunate facet) anterior to the flat volar surface of the distal radius and was approximately $5 \mathrm{~mm}$ thick. Their findings may explain its relative vulnerability to injury and the difficulty encountered when trying to secure it with a plate and screws.

To avoid complications in the VLP fixation, we propose safe and secure fixation area for the plate in standard surgical procedures based on the volar distal radius anatomy. The first step is to explore the intermediate fibrous zone and the distal part of pronator quadratus muscle at the volar distal radius precisely to visualize the exact plate placement. Anatomic reduction of the volar cortex, which facilitates restoration of the radial length, inclination, and volar tilt, is the second step. It is important not to leave the dorsal and rotational deformities.
The third step is to place the distal edge of the VLP to the volar surface of the radius proximal to the watershed line and without any interval between the plate and the radius. The fourth step is the precise drilling and maneuvering of screws, followed by checking their reduction and position of plate and screws by fluoroscopy or arthroscopy. The final step includes the coverage of the distal part of VLP by soft tissues, including the intermediate fibrous zone and the pronator quadratus, to reconstruct the smooth gliding surface of the flexor tendons.

\section{Note}

No benefits in any form have been received or will be received related directly or indirectly to the subject of this article.

\section{Conflict of Interest}

None.

\section{References}

1 Windisch G, Grechenig W, Peicha G, Tesch NP, Seibert FJ. Capsular attachment to the distal radius for extracapsular placement of pins. Surg Radiol Anat 2001;23(05):313-316

2 Windisch G, Clement H, Tanzer K, et al. Promontory of radius: a new anatomical description on the distal radius. Surg Radiol Anat 2007;29(08):629-633

3 Orbay JL, Touhami A. Current concepts in volar fixed-angle fixation of unstable distal radius fractures. Clin Orthop Relat Res 2006;445:58-67

4 Gasse N, Lepage D, Pem R, et al. Anatomical and radiological study applied to distal radius surgery. Surg Radiol Anat 2011;33(06): 485-490

5 Imatani J, Akita K, Yamaguchi K, Shimizu H, Kondou H, Ozaki T. An anatomical study of the watershed line on the volar, distal aspect of the radius: implications for plate placement and avoidance of tendon ruptures. J Hand Surg Am 2012;37(08):1550-1554

6 Omokawa S, Abe Y, Imatani J, et al. Treatment of intra-articular distal radius fractures. Hand Clin 2017; In press

7 Shimizu H, Imatani J, Akita K, et al. Positional relationship between the distal radius and the flexor tendons [in Japanese]. J Jpn Socie Surg Hand 2010;27:587-589

8 Kondo H, Imatani J, Moritani S, et al. Multidetector computed tomography investigation of the path of the flexor pollicis longus tendon: avoiding flexor tendinopathy following volar locking plate fixation [in Japanese]. J Jpn Socie Surg Hand 2012;29:22-25

9 Limthongthang R, Bachoura A, Jacoby SM, Osterman AL. Distal radius volar locking plate design and associated vulnerability of the flexor pollicis longus. J Hand Surg Am 2014;39(05):852-860

10 Oura K, Oka K, Kawanishi Y, Sugamoto K, Yoshikawa H, Murase T. Volar morphology of the distal radius in axial planes: a quantitative analysis. J Orthop Res 2015;33(04):496-503

11 Yoneda H, Iwatsuki K, Hara T, Kurimoto S, Yamamoto M, Hirata H. Interindividual anatomical variations affect the plate-to-bone fit during osteosynthesis of distal radius fractures. J Orthop Res 2016;34(06):953-960

12 Pichler W, Clement H, Hausleitner L, Tanzer K, Tesch NP, Grechenig W. Various circular arc radii of the distal volar radius and the implications on volar plate osteosynthesis. Orthopedics 2008; 31(12):1-4

13 Andermahr J, Lozano-Calderon S, Trafton T, Crisco JJ, Ring D. The volar extension of the lunate facet of the distal radius: a quantitative anatomic study. J Hand Surg Am 2006;31(06):892-895 